\title{
System Safety Analysis and Fire Prevention Strategy of Public Entertainment Places
}

\author{
Shifeng Ding ${ }^{1, *}$, Nana Ding ${ }^{2}$ \\ ${ }^{1}$ Chaoyang District Fire and Rescue Section, 130012, Changchun, Jilin Province, China \\ ${ }^{2}$ The First Project Agent Construction Management Office of Logistic Support Forces of the Chinese People's Liberation Army, 210003, \\ Nanjing, Jiangsu Province, China
}

\begin{abstract}
With the accelerated pace of Chinese economic development in recent years, the public entertainment industry is constantly developing, and the corresponding fire accidents are also on the rise, posing a huge threat to people's lives and property. There are a lot of people gathering in public entertainment places, and it is difficult to evacuate people when a fire accident occurs. It is one of the key fire protection management areas. Fires in public entertainment places cause immeasurable losses to the lives and property safety of people. How to strengthen the fire protection work of public entertainment places is the key task of the fire management department. Based on the accident tree analysis method, this paper conducts a systematic safety analysis of the public entertainment industry and its typical fire cases, and concludes that the decoration of flammable and combustible materials and the storage of flammable and explosive materials are the main causes of fires in public entertainment places, and proposes fire prevention strategies.
\end{abstract}

\section{Introduction}

In recent years, the improvement of living standards in China has promoted the vigorous development of the entertainment industry. Public entertainment places are increasing year by year, and they have become important places where the people are most willing to gather, play and relax. The place is crowded with people and lacks vigilance to potential safety hazards around them in a state of rest and relaxation. Therefore, once a fire occurs, it will cause a large number of people to evacuate difficult, resulting in serious casualties. On the one hand, it is due to the negligence of the management staff. On the other hand, it has a great relationship with the design and decoration of the fire prevention strategy of these places. To a large extent, some unreasonable decoration designs have contributed to the occurrence and spread of fires, and have further expanded the loss of our lives and properties. Therefore, it is very important to strengthen the research of fire protection countermeasures in public places.

More and more public entertainment venues have become gathering places for people. For example, saunas, theaters, hospitals, exhibition halls, stations and wharfs, restaurants, shopping malls, libraries, cultural palaces, youth palaces, children's palaces, clubs, dance halls, etc., are all places where people are concentrated and flowed. However, these places have many safety hazards. On December 30, 2004, a nightclub in Buenos Aires, the capital of Argentina, caused a fire after setting off fireworks, causing 194 deaths and 1432 injuries. On September 20, 2008, a fire broke out in the Dance King Club in Longgang District, Shenzhen, which eventually caused 44 deaths and 64 injuries. Although losses are rare, there are lessons for the same place. On January 31, 2009, a bar in the urban area of Changle, Fujian Province, China caused a fire when guests set off fireworks on the table, causing 15 deaths and 24 injuries. Abroad, similar accidents have also appeared in endlessly. On January 1, 2009, a fire broke out in a nightclub in Bangkok, the capital of Thailand, at midnight of the New Year, killing at least 62 people and injuring more than 200 others. Work safety accidents, including fires and explosions, cannot be solved by one or two publicity and education and campaign-style surprise inspections and rectifications. There are profound and complex reasons behind the accident. The gestation, development and outbreak of accidents are the evolution of systems composed of different factors such as people, things, and the environment. Therefore, the system safety perspective should be adopted to analyze the deep-seated causes of accidents, and the method of accident cause theory should be used to predict and prevent accidents.

\section{Accident-causing theory}

Accident-causing theories includes the theory of frequent accidents, causality, energy transfer theory, disturbance origin theory, trajectory intersection theory, etc. Most safety experts in my country believe that accidents are not caused by a single factor, nor are they caused by accidental personal errors or simple equipment failures, but the result of a combination of various factors. Accidents are random events with complex causes, but they also have their inevitable statistical laws. They are the result of accidental events triggering various hazards in society, management, and production. The fact of the accident is that the cause and the perpetrator accidentally triggered the disaster phenomenon formed by the harm and the victim.

Accidental events are triggered because of the existence of various hidden dangers (unsafe state of objects) and human errors (unsafe behaviors) of the environmental conditions in production, which together constitute the direct cause of the

*Corresponding author: 806781836@qq.com 
accident. The process of accidents can be expressed as "management factors" from the basic causes of "social factors", and further "risk factors in production". Casualties and losses occur through accidental factors of people and things. The process of investigating and analyzing accidents is the opposite of the above experience, such as reverse tracking. The comprehensive accident model provides clear ideas for the comprehensive identification of various hazards and the control of accidents through a variety of means and methods. It is especially suitable for the analysis of the cause of serious fire accidents. The model is shown in Figure 1.

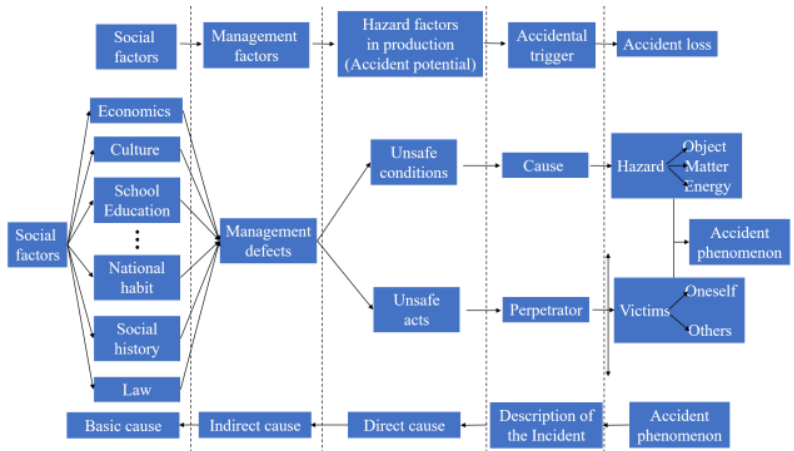

Figure 1. Comprehensive theory of accident model.

\section{3 "9.20" Shenzhen Dance King Club fire accident analysis}

At about 23:00 on September 20, 2008, a huge fire broke out in the Dance King Club, Longdong Community, Longgang Street, Longgang District, Shenzhen. After the fire department of Longgang District put out the fire, the fire was quickly extinguished. The accident caused 43 deaths and 88 people. Injured, 51 of them need to be hospitalized. After the accident, the State Council established an accident investigation team and invited the Supreme People's Procuratorate to participate in the investigation. The Supreme People's Procuratorate also set up a Shenzhen investigation team to simultaneously intervene in the investigation of suspected duty crimes in the accident. It was determined that the nature of the accident was a safety production liability accident during the operation process.

\subsection{Accident phenomena and consequences}

The fire took place on the 3-story dance hall stage with a construction area of about 700 square meters. At that time, there were about 500 spectators in the auditorium. The cause of the fire was to set off fireworks on the stage to ignite the ceiling. At that time, after the ceiling was ignited, the staff knew there was a fire, but they did not say anything, nor did they organize the evacuation of the audience. Instead, they used fire extinguishers to extinguish the fire. The fire then spread to the auditorium, causing people to panic and flooding into narrow staircase openings and evacuation passages. Due to the use of a large number of combustible materials during the decoration process, the fire spread rapidly and a lot of dense smoke appeared. At this time, the power supply was interrupted at the scene, and the personnel escaped and lost their direction. In addition, the outer windows of buildings are often closed, which greatly increases the difficulty of rescue. In the end, 43 people were killed and 64 people were injured. The course of the accident is not complicated, but the consequences of the accident (that is, the accident phenomenon in the synthesis theory) are extremely serious.

(1) Harmful substances: high-temperature toxic smoke produced by the burning of combustibles in a fire. The dance king club uses a large number of sound-absorbing sponges for decoration. The sponges are polyurethane synthetic materials with low ignition point, high smoke and strong combustion products.

(2) Victim: the audience in the audience hall.

(3) Causes: combustibles on the top of the stage (polyurethane foam, polystyrene sandwich panels).

(4) Perpetrators: business managers, safety supervisors, site staff, architectural designers, construction builders and supervisors.

(5) Incidental event that triggers the accident: fireworks are set off during the show.

\subsection{Direct cause of accident}

The direct cause of the accident was that the funny sketch actors set off fireworks during the performance, which ignited the combustible ceiling. Due to the use of combustible materials during decoration, the fire spread very quickly.

The main reasons for the heavy casualties are that after the fire, there are too many combustible materials, and there is no automatic sprinkler system, and the fire extinguisher expires, causing the fire to spread rapidly from the fire. Polyurethane foam, polystyrene foam and sound-absorbing sponge Largearea burning of other materials produces high-temperature and toxic fumes; second, the escape passage in the building is complicated, and a large number of exterior windows of the building are blocked, and the on-site power outage makes it impossible for people to escape in time when a fire occurs; third, there is no alarm device. The staff knew about the fire late, and the staff who first discovered the fire did not organize the evacuation of the audience in time, so many people lost the best opportunity to escape; the site did not conduct safety training for employees, did not organize emergency evacuation drills, and employees lacked escape and self-help Help each other knowledge and ability. The unsafe state of the analyte and the unsafe behavior of people from the direct causes identified above are as follows:

(1) Object unsafe acts: the use of combustible materials for decoration does not meet the requirements of GB50222-2017 "Code for fire protection in design of interior decoration of buildings"; there is no automatic sprinkler system, which does not meet the requirements of GB50016-2018 "Code for fire protection design of buildings"; fire extinguishers Expired, lack of fire-fighting power supply equipment and emergency lighting is not installed, fire detection and alarm devices do not meet the requirements of GB 50116-2013 "Code for design of automatic fire alarm system";.

(2) Human unsafe behavior: fireworks are set off indoors in violation of regulations; employees are not given safety education and training, most employees lack the knowledge and ability to escape, self-rescue, and mutual rescue, and they are unable to organize the evacuation of trapped persons; illegal blockade Building exterior windows.

The unsafe state of these objects and unsafe behavior of people are hidden dangers of accidents.

\subsection{Indirect cause}

The indirect causes of the "September 20" fire accident are as follows: first, the main responsibility for the safety operation of the site has not been implemented; second, the public security fire department is ineffective in performing fire supervision and management responsibilities; third, the construction department is in the construction of the project There is a serious lack of supervision; fourth, the safety supervision department does not perform the comprehensive supervision 
responsibilities of safety production in place; fifth, the implementation of the safety supervision responsibilities of local governments is not effective. In summary, these five aspects are essentially management issues, including internal enterprise management and government administration. These management deficiencies are the root cause of the accident.

\subsection{Basic cause}

According to the comprehensive theory of accident cause, the indirect cause comes from the basic cause, and many social factors constitute the basic cause, including economic, cultural, educational, legal, historical and ethnic customs, etc., which lead to the lack of management.

The first is cultural reasons. Many units do not attach importance to the construction of a safety culture, thinking that as long as there are no problems, the word "safety" has nothing to do with them. This has led to a lack of investment in safety management, and safety hazards have not been eliminated, but have increased day by day. Regarding fire safety in many enterprises and business premises, fire safety work is a mere formality, just to cope with inspections, and there is no safety culture at all. Culture is a kind of belief, a concentrated expression of the inner character of a nation. Therefore, cultural construction requires unremitting efforts from generations of people, but the power of culture is the key. Once it is formed, it will play an important role in people's daily life and work, as well as fire safety culture. On the contrary, many large enterprises engaged in the production, storage and transportation of flammable and explosive dangerous goods attach great importance to the construction of corporate safety culture. They use excellent corporate safety culture to correct unsafe conditions and unsafe behaviors of people in a timely manner. So there are few safety accidents. Therefore, the power of culture may seem intangible, but it is infinite.

The second is legal reasons. Although in the new era, the legal awareness of the whole society has improved, a considerable number of people still have weak legal concepts. For managers, some people do not pay attention to laws and regulations related to safe production. For law enforcement officials, there are occasions when law enforcement is not abiding by law, law enforcement is not strict, and violations are not investigated. The restriction of laws and regulations is the last line of defense for safe production and safe operation. However, some companies failed to implement the laws and regulations that should have been implemented, and the relevant departments did not perform their supervision responsibilities, and the hidden dangers of accidents became more serious.

\section{Dangerous factors in fire}

A large amount of heat and smoke (including general hot smoke and toxic and harmful gases) will be released during the development of a fire. When people in public entertainment places see that the environment they usually adapt to becomes completely unrecognizable due to the fire, they will inevitably have fear psychological. The temperature, smoke layer, and toxic gases in the fire products will have a great impact on the physiology and psychology of the personnel in the fire scene, thereby affecting the choice of evacuation routes and evacuation preparation time, and ultimately affecting the evacuation efficiency.

\subsection{High temperature and heat radiation}

The high temperature generated in a fire will physically make people in the fire scene feel hot, dizzy, and physically collapsed, and psychologically will make people in the fire scene feel very nervous, flustered, frightened and uneasy, thereby forcing people to take measures. Avoid the high temperature. If there is no way to evacuate in the fire scene, they will generally choose to retreat to a corner with a lower temperature to temporarily avoid the high temperature. Therefore, most of the besieged persons found in the fire scene were mostly in the corners, in the toilets, and under the beds.

\subsection{Toxic fumes}

Fire smoke can be defined as solid particles, liquid droplets and gas phase products produced when fuel is decomposed or burned. Generally speaking, it is the smoke that poses a real threat to the lives of people in fires. The survey found that the rate of deaths due to inhalation of harmful combustion products in fires is much higher than that of other injuries. The strong smoke made people unbearable at first, breathing difficulties, unable to open their eyes, physical harm, from slight poisoning to deep poisoning, reduced consciousness, and finally loss of consciousness and death. Fire smoke can also cause insufficient oxygen supply to the human brain, resulting in decreased thinking ability, slow response, and decreased memory and judgment. For example, misjudge the evacuation direction, can't remember the location of the safety exit, run aimlessly, grab the door frame instead of twisting the handle at the exit, return to the smoke area after escaping from the smoke area, etc. Generally speaking, as the concentration of smoke increases, the memory and judgment of the victims almost plummet. Therefore, when the smoke is thick, it is difficult to find a safe exit unless people who are very familiar with evacuation routes and those who know the location and direction of the evacuation exit in advance. Modern building materials and decoration materials use a large number of high molecular polymers, which release a lot of toxic and harmful components under fire conditions, which pose a huge threat to the lives of personnel. The data listed in Table 1 reflects the impact of CO concentration and cumulative dose on personnel status.

Table 1. The influence of $\mathrm{CO}$ concentration and cumulative dose on personnel status.

\begin{tabular}{c|c|c|c}
\hline $\mathrm{C}(\%)$ & $\begin{array}{c}\text { Exposure } \\
\text { time(min) }\end{array}$ & $\begin{array}{c}\text { Accumulated } \\
\text { dose } \\
(\% \mathrm{~min})\end{array}$ & $\begin{array}{c}\text { Staff } \\
\text { response }\end{array}$ \\
\hline 0.02 & $120 \sim 180$ & $2.4 \sim 3.6$ & $\begin{array}{c}\text { Moderate } \\
\text { headaches }\end{array}$ \\
\hline 0.08 & 45 & 3.6 & $\begin{array}{c}\text { Moderate } \\
\text { headaches }\end{array}$ \\
\hline 0.32 & $10 \sim 15$ & $3.2 \sim 4.8$ & Dizzy \\
\hline 0.32 & 30 & 9.6 & $\begin{array}{c}\text { Possible } \\
\text { death }\end{array}$ \\
\hline 0.69 & $1 \sim 2$ & $0.69 \sim 1.38$ & Dizzy \\
\hline 1.28 & $\begin{array}{c}0.1(2 \sim 3 \text { deep } \\
\text { breaths })\end{array}$ & 0.128 & $\begin{array}{c}\text { Lose } \\
\text { consciousness }\end{array}$ \\
\hline 1.28 & $1 \sim 3$ & $1.28 \sim 3.84$ & Death \\
\hline
\end{tabular}




\section{Fire protection strategy}

Since my country's reform and opening up, my country's national economy has continued to improve rapidly, especially since joining the WTO, people's quality of life has rapidly improved. At the same time, people are not only satisfied with food and clothing, more people begin to focus on spiritual life and quality of life, leisure and entertainment and other places have gradually increased, and their forms have begun to develop in a diversified direction. The increase of these places not only enriches the daily lives of our people, but also improves the quality of people's daily lives. However, because of the unreasonable decoration design and fire protection strategy of these places, the negligent management and supervision of the managers, and the weak awareness of fire protection among the people, there have been many accidents and casualties. Based on the analysis of accident tree, this paper makes a systematic safety analysis of the public entertainment industry and its typical fire cases, and concludes that it is very important to strengthen the fire prevention countermeasures of public entertainment places.

(1) Strengthen the selection and management of decoration materials. Choose scientific decoration materials, ensure that the decoration materials used in the place are non-combustible materials, and ensure that the combustion of these materials will not release toxic fumes even if a fire occurs. At the same time, the selection of electrical wiring and equipment applications in the site should be very careful, and professionals should be regularly inspected.

(2) Set up complete fire fighting equipment. Public entertainment places are areas prone to fire, so it is very important to set up complete fire fighting equipment and do a good job of fire fighting.

(3) Strictly implement the safety responsibility system. The safety responsibility system is a powerful countermeasure to ensure fire safety, and the effective implementation of relevant responsibilities to specific personnel can promote more effective fire protection work. At the same time, regular fire drills are carried out to ensure that every employee participates and improve the overall fire protection awareness.

(4) Strengthen personnel fire safety training. It is necessary to conduct regular fire-fighting knowledge training for employees, enhance their fire-fighting awareness, and promote the effective implementation of the fire safety responsibility system to each individual.

\section{Conclusion}

This article is based on the accident tree analysis method to conduct a systematic safety analysis of the public entertainment industry and its typical fire cases. It is concluded that the decoration of flammable and combustible materials and the storage of flammable and explosive materials are the main reasons for fires in public entertainment places. The above puts forward specific four aspects of fire prevention strategy, strengthen fire prevention and fire prevention, and comprehensively improve fire prevention efficiency. Supervise and inspect the decoration materials used in public entertainment places. A complete set of fire-fighting equipment in public entertainment places shall be installed to ensure timely and effective fire fighting in the event of a fire. In addition, the implementation of the fire safety responsibility system for public entertainment venues and personnel fire safety training have played a positive role in the fire protection of public entertainment venues.

\section{References}

1. Jingze, Z., Yanxin, L. (2019) Problems and Countermeasures in Fire Protection Supervision of Public Entertainment Places. Urbanism and Architecture. 17: 197-198.

2. Xiaopeng, C. (2011) Analysis of Fire Hazard in Public Entertainment Occupancies and Fire Prevention and Control Measures. Science \& Technology for Development. S1: 22-23.

3. Rong, Q., Dongzhi, P. (2005) Discuss of the AccidentCausing Theory. 3: 1-10.

4. Tengteng, L., Huidong, W., Qiuhua, W., et al. (2011) Research on model building with high-rise building fire accident-causing theory. Journal of Safety ence and Technology. 5: 16-20.

5. Guangjian, Z., Xiaojiang, D., Wei, L. (2007) Fault Tree Analysis of Fire Accident of Large-scale Hotel. Safety and Environmental Engineering. 4: 83-85.

6. Code for fire protection in design of interior decoration of buildings (GB50222-2017) [S].

7. Code for fire protection design of buildings (GB500162018) [S].

8. Code for design of automatic fire alarm system (GB 50116-2013) [S]. 